\title{
Evolution of $\gamma$-Vibrations at High Spin
}

\author{
J F Sharpey-Schafer ${ }^{1}$
}

University of Western Cape

Department of Physics, P/B X17, Bellville, ZA-7535 South Africa.

E-mail: jfssetlabs.ac.za

\section{S N T Majola*, R A Barkף, S P Bvumbi®, T S Dinoko§, E A Lawrieףl, J J Lawrieףl, S} M MullinsףI, J N Orce $\$$ and P Papka\#

§Universities of Western Cape, $\mathbf{N}$ Johannesburg, *Cape Town and \#Stellenbosch. IiThemba Laboratory for Accelerator Based Sciences

PO Box 722, Somerset-West, ZA-7129 South Africa

\begin{abstract}
Intrinsic collective excitations in deformed nuclei [1] are $\boldsymbol{\gamma}\left(\mathrm{Y}_{2,2}\right)$ and Octupole $\left(\mathrm{Y}_{3, \mu}\right)$ vibrations; $\boldsymbol{\beta}\left(\mathrm{Y}_{2,0}\right)$ vibrations are at energies above the pairing gap [2,3] and have not been observed. In the past, experiments on rotating deformed intrinsic structures to populate levels at high spin have been an effective method of revealing details of the underlying microscopic configurations. Not many $\gamma$-bands have been extended to high spin due to their distance from the yrast line. However, Coulomb excitation experiments and the use of the very large $\gamma$-ray detector arrays have enabled the $\boldsymbol{\gamma}$-bands in several nuclei to be extended to much higher spins than previously achieved. We report on recent data on $\gamma$-bands at high spin and on the properties of $\gamma$-bands in general.
\end{abstract}

XLIX International Winter Meeting on Nuclear Physics- BORMIO2011

Bormio, Italy

January 24-28 2011

$1 \quad$ Speaker 


\section{Introduction}

The underlying microscopic structure of $\boldsymbol{\gamma}$-vibrations of deformed nuclei has, for a long time, been of great interest with descriptions of this collective degree of freedom ranging from traditional strong coupling models to quasiparticle-phonon models to IBA and other symmetry representations. The traditional interpretation [1] of the lowest $K^{\pi}=2^{+}$rotational bands, that consistently appear in every even-even deformed nucleus, has been that they are a vibration in the $\gamma$ degree of freedom. Quantum mechanical liquid drop models, that start with the 5 dimensional Bohr Hamiltonian [4-6], include the $\beta$ and $\gamma$ degrees of freedom and give, in an obvious notation, the energies of collective states as $[7,8]$;

$$
E_{x}\left(n_{\beta} n_{\gamma} I K\right)=\hbar \omega_{\beta}\left(n_{\beta}+1 / 2\right)+\hbar \omega_{\gamma}\left(2 n_{\gamma}+1 / 2|K|+1\right)+\left[I(I+1)-K^{2}\right] \hbar^{2} / 2 \mathscr{I}
$$

Most text books manage to miss the $1 / 2|\boldsymbol{K}|$ term in Equ. (1), giving the impression that the first $K^{\pi}=2^{+}$band has $\boldsymbol{n}_{\gamma}=1$. But Equ. (1) shows that the traditional $K^{\pi}=2^{+} \gamma$-band is not a band containing a quantum in the $\gamma$ direction but has $\boldsymbol{n}_{\gamma}=\mathbf{0}$ and a bandhead excitation energy given by $\boldsymbol{E}_{x}=\boldsymbol{\hbar} \boldsymbol{\omega}_{\gamma}+\boldsymbol{\hbar}^{2} / \mathscr{T}$. In the rotation-vibration model there is a strong coupling between rotations and $\gamma$-vibrations, physically expressing the fact that rotations with non-vanishing $\mathrm{K}$ become possible only in the presence of dynamical triaxiality [8]. Any model having the $\gamma$ degree of freedom will have zero-point fluctuations and a similar origin for $\mathrm{K}=2^{+}$bands.

In contrast, early microscopic models of collective vibrations in deformed nuclei [9-13] assume the existence of a vibrational "phonon" or "boson". They then have to construct this entity out of some set of basis states. This they do by postulating an interaction, expanding their collective phonon in a truncation of this basis and then using the variation principle to minimise the phonon energy in terms of their interaction parameters. Needless to say an industry is created [14-18] discussing optimisation of bases, truncations and "fitting" the parameters! One of the many difficulties with this approach is that, without exception, it is assumed that the lowest excited $\mathrm{O}_{2}{ }^{+}$states in deformed nuclei are $\beta$-vibrations, which they are not. Indeed the experimental evidence (see refs. [2] and [3] and references therein) suggests that $\beta$-vibrations lie above the pairing gap and have not been observed so far. Phenomenological models, such as the IBA [19] and geometric approaches [20] suffer from similar difficulties plus the disadvantage that they say nothing at all about the underlying microscopic configurations.

RPA calculations seem to do rather better [21-24]. The recent Triaxial Projected Shell Model (TPSM) calculations [25-29] seem to give a real hope of obtaining a clear microscopic and physically accurate picture of $\boldsymbol{\gamma}$-vibrations of deformed nuclei. Reference [28] gives a very clear history of approaches to descriptions of $\gamma$-vibrations.

It is very clear that the $\gamma$ degree of freedom, in describing the shapes of deformed nuclei, is indispensible. A nice illustration of this is the self-consistent relativistic mean field plus BCS calculations of the München group and colleagues [30,31]. For the deformed nuclei ${ }^{148} \mathrm{Nd}$ and ${ }^{150} \mathrm{Nd}$, strong minima with oblate shapes seen in the calculations using only $\beta$ deformation [30], turn out to be saddle points on a very $\gamma$-soft total energy surface when the $\gamma$ degree of freedom is included in the calculations [31]. 
In this contribution we briefly review the experimental data and properties of known $\gamma$ bands and present some recent data on bands observed at higher spins than normally achieved.

\section{Transfer reactions to $\gamma$-bands}

In looking for clues to the underlying microscopic structure of any nuclear states, it is usual to examine the one and two particle transfer strengths in direct reactions that populate these states. Two particle transfers, e.g. by $(\mathrm{p}, \mathrm{t})$ and $(\mathrm{t}, \mathrm{p})$ reactions from the target ground $0^{+}$ state of an even-even nucleus, will populate the $2_{\gamma}{ }^{+}$band head with an $\boldsymbol{L}=\mathbf{2}$ transition but give no information on the single particle orbits involved, other than how much pairs of neutrons (or protons) are involved in the wavefunction. Single particle direct reactions to a $\gamma$-band, e.g. $(\mathrm{d}, \mathrm{p}),(\mathrm{p}, \mathrm{d}),\left({ }^{3} \mathrm{He}, \mathrm{d}\right),\left(\mathrm{d},{ }^{3} \mathrm{He}\right)$, give considerable information as the spin/parity of the odd target nucleus and the transferred nucleon must add vectorally to the final state spin. As pairing is an important feature of deformed nuclei, a first order description of their properties is given by introducing pairing interactions in the Nilsson basis. Hence single particle transfer gives information on the quasi-particle/hole structure of any states not in the ground state band of the final nucleus. States that are strongly populated will consist of the target odd quasi-particle coupled to some other quasi-particle. As $\boldsymbol{K}$ is a good quantum number for axially symmetric states, any p-h component of the $\gamma$-band configuration should be composed of quasi-particles in Nilsson orbits $\left[\boldsymbol{N} \boldsymbol{n}_{z} \boldsymbol{\Lambda}\right] \boldsymbol{\Omega}$ of the same parity and where $\boldsymbol{\Delta} \boldsymbol{K}=\left|\boldsymbol{\Omega}_{\text {target }} \pm \boldsymbol{\Omega}_{\text {transfer }}\right|=\mathbf{2}[32,33]$.

\subsection{Neutron Transfer}

The (d,p) reaction has been used to populate states in ${ }^{158} \mathrm{Gd},{ }^{164} \mathrm{Dy},{ }^{172} \mathrm{Yb}$ and ${ }^{173} \mathrm{Yb}$ [32]. A straightforward calculation using Nilsson wavefunctions and the assumptions of [11] gives a good account of the relative strengths of the relative populations for the ground state and $\gamma$ bands in all four nuclei and the configurations involved have $\boldsymbol{\Delta K}=2$. Similarly the

${ }^{151} \mathrm{Sm}(\mathrm{d}, \mathrm{p}){ }^{152} \mathrm{Sm}$ reaction [34] strongly populates the $\gamma$-band as the ground state of ${ }^{151} \mathrm{Sm}$ is [523]5/2 and the [521]1/2 orbital, giving $\boldsymbol{\Delta K}=2$, is available above the Fermi Surface. In contrast, the neutron pick-up reaction ${ }^{151} \mathrm{Sm}(\mathrm{p}, \mathrm{d}){ }^{150} \mathrm{Sm}$ does not populate the $\gamma$-band [34] as there is no suitable $\boldsymbol{\Delta} \boldsymbol{K}=\mathbf{2}$ orbital to couple to below the Fermi Surface.

\subsection{Proton Transfer}

Proton stripping reactions to ${ }^{154} \mathrm{Gd}$ using the $\left({ }^{3} \mathrm{He}, \mathrm{d}\right)$ and $(\alpha, \mathrm{t})$ reactions [35] populate the $\gamma$-band very strongly. The target nucleus ${ }^{153} \mathrm{Eu}$ has its odd proton in the $[431] 5 / 2^{+}$orbit and the $\Delta K=\mathbf{2}$ orbit $[411] 1 / 2^{+}$is just above the Fermi Surface. Again, in contrast, the $(\mathrm{t}, \alpha)$ proton pickup reaction to the nuclei ${ }^{152} \mathrm{Sm} \mathrm{[36],}{ }^{164} \mathrm{Dy}[37]$ and ${ }^{174} \mathrm{Yb}$ [38] do not populate the $\boldsymbol{\gamma}$-band at all. Again this is because there are no suitable $\boldsymbol{\Delta} \boldsymbol{K}=\mathbf{2}$ orbitals below the Fermi Surface.

\subsection{Two Neutron Transfer}

Two neutron transfer reactions only populate natural parity states in even-even nuclei as the angular momentum transferred in the reaction $\boldsymbol{L}$ determines both the spin and the parity of the final state. The $(\mathrm{p}, \mathrm{t})$ pick-up reactions usually populate the $\boldsymbol{\gamma}$-band very weakly, for instance in the Gd isotopes [39,40] and $\mathrm{W}$ isotopes [41]. The two neutron pick-up to the ${ }^{168} \mathrm{Er} \gamma$-band 
[42] is stronger than most at about $15 \%$ of the intensity to the ground state. The $\gamma$-bands can also be clearly identified in the two neutron pick-up to ${ }^{228,230} \mathrm{Th}$ and ${ }^{232} \mathrm{U}$ [43]. The (t,p) reaction often has no sign at all of the $\gamma$-band in even-even nuclei, as in the famous paper by Casten et al. [44] and as shown in Fig. 1 for the ${ }^{152} \mathrm{Gd}(\mathrm{t}, \mathrm{p}){ }^{154} \mathrm{Gd}$ reaction [45]. No transfer to the $\gamma$-bands is also observed in the $(t, p)$ reaction to the even Sm isotopes [46].

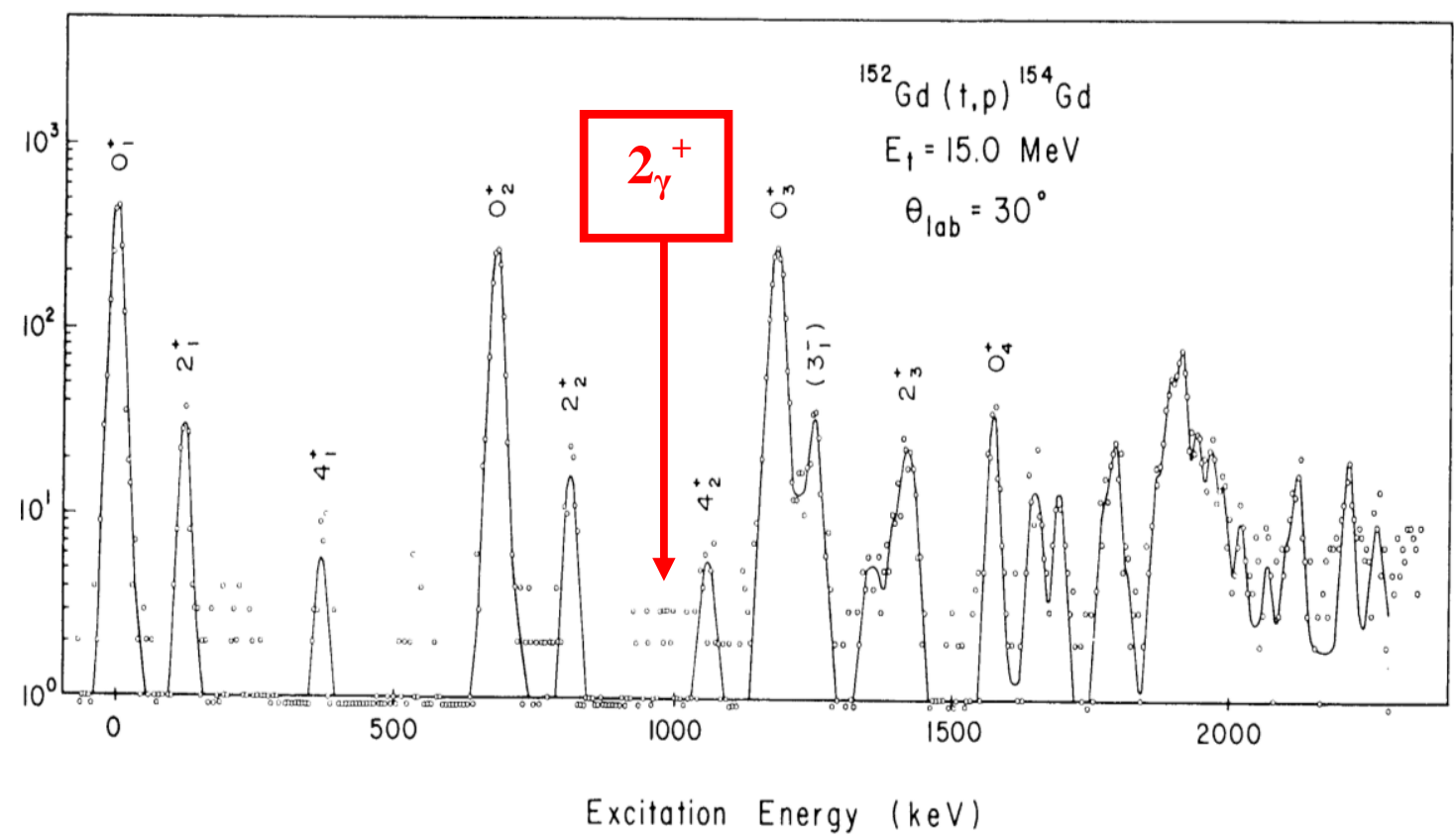

Figure 1: Proton spectrum from the ${ }^{152} \mathrm{Gd}(\mathrm{t}, \mathrm{p}){ }^{154} \mathrm{Gd}$ reaction [45] showing that the $\mathbf{2}_{\gamma}{ }^{+}$level is not populated in the two neutron transfer reaction.

\section{Systematics of $\gamma$-bands}

A partial decay scheme showing positive parity levels observed [3] in the prolate deformed nucleus ${ }^{154} \mathrm{Gd}$ using $(\alpha, \mathrm{xn})$ reactions is shown in Fig. 2. The levels to the right are a $\gamma$ band based on the ground state intrinsic state. The levels are divided into even $2^{+}, 4^{+}, 6^{+} \ldots$ and odd $3^{+}, 5^{+}, 7^{+} \ldots$ spin levels for clarity. The even spin levels decay to the levels in the ground state band (gsb) by not only $\boldsymbol{\Delta} \boldsymbol{J}=\mathbf{2}$ transitions, but also $\boldsymbol{\Delta} \boldsymbol{J}=\boldsymbol{0}$ and $\boldsymbol{\Delta} \boldsymbol{J}=\mathbf{- 2}$ transitions. As $\boldsymbol{A} \boldsymbol{K}=$ $\boldsymbol{2}$ in these out-of-band transitions, M1 components are $\boldsymbol{K}$-forbidden in the $\boldsymbol{\Delta J}=\boldsymbol{0} \boldsymbol{\gamma}$-rays. Similarly the $\boldsymbol{\Delta J}= \pm \boldsymbol{1}$ transitions from the odd spin members to the gsb will be mostly E2 and contain very small M1 components at most [47,48].

Generally in-band $\boldsymbol{\Delta J}=\boldsymbol{1}$ transitions between the even and odd spin members of $\boldsymbol{\gamma}$-bands are very weak. This means that $\boldsymbol{g}_{K} \approx \boldsymbol{g}_{\boldsymbol{R}}$ for $\boldsymbol{\gamma}$-bands.

Equation (1) may be used to calculate $\hbar \omega_{\gamma}$ for a series of nuclei from the excitation energies of their $\gamma$-bands and their moments-of-inertia. These are shown in Fig. 3 for deformed nuclei with $Z=60$ to 70 . It can be seen that values of $\hbar \omega_{\gamma}$ lie mainly between 750 and 1100 $\mathrm{keV}$. 


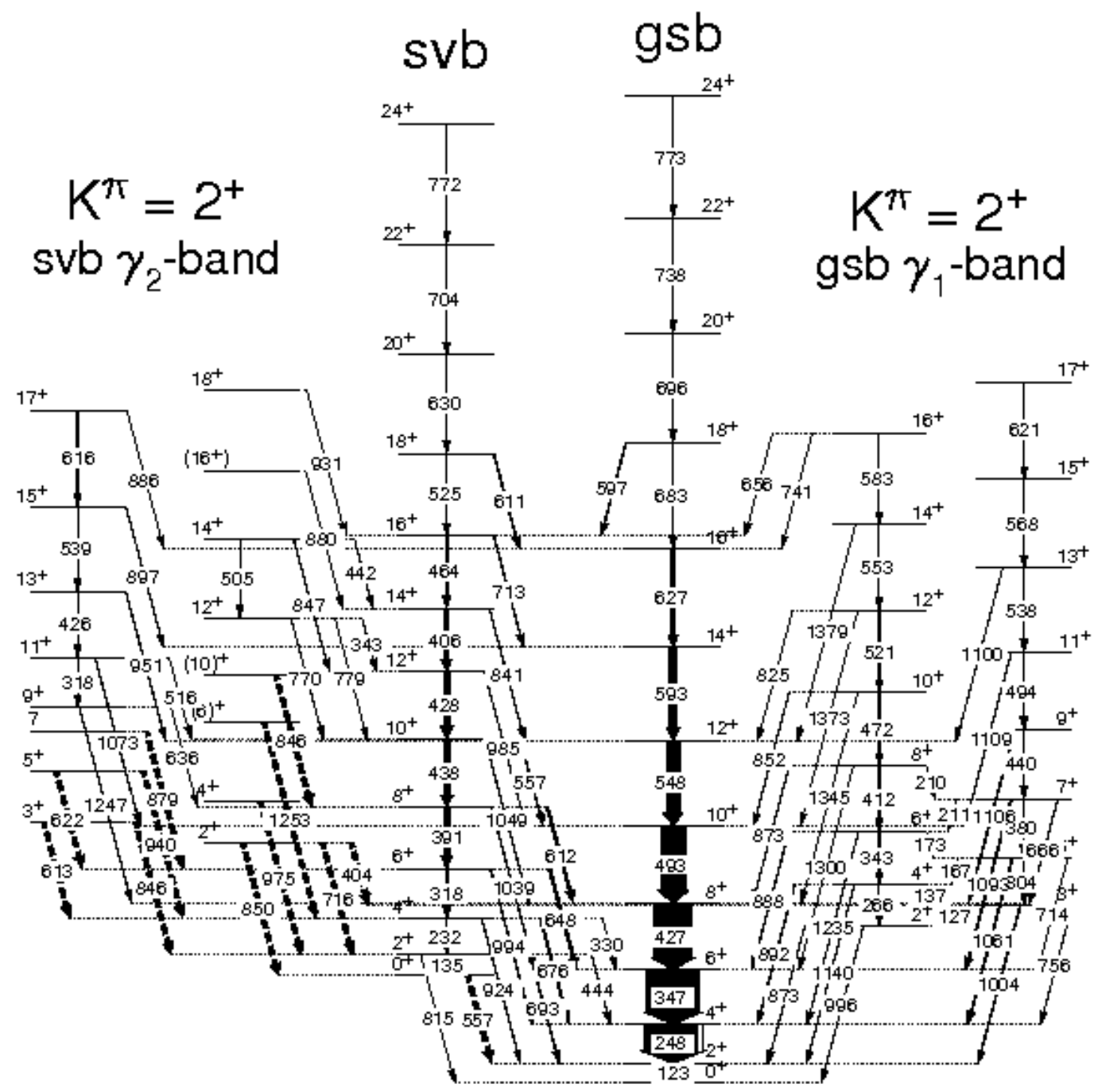

Figure 2: Partial decay scheme for ${ }^{154} \mathrm{Gd}$ showing positive parity bands in $(\alpha, \mathrm{xn})$ experiments [3]. The widths of the $\gamma$-rays are proportional to the intensities found in the $(\alpha, 4 n)$ experiment. The $\gamma$-rays shown as dashed arrows were only observed in the $(\alpha, 2 n)$ experiment. The ground state band $\left|\mathbf{0}_{1}{ }^{+}\right\rangle$is labeled $\mathbf{g s b}$, the second vacuum band $\left|\mathbf{0}_{2}{ }^{+}\right\rangle$is labeled svb. Each has its own $\boldsymbol{K}^{\pi}=2^{+} \gamma$-band.

It is not very usual for $\gamma$-bands to be identified much above spin $12^{+}$as they are usually about $1.0 \mathrm{MeV}$ above the yrast line. This makes it difficult to populate such states in fusionevaporation (HI,xn) reactions as they are embedded in other structures which compete for intensity. The use of very heavy ion beams to Coulomb excite the most deformed nuclei has, in favourable cases, allowed $\gamma$-bands to be traced to much higher spins. In table 1 we list some even-even nuclei in which the $\gamma$-band has been observed above $15^{+}$, giving both the reaction used and the highest spin reached both for even and odd spins. 


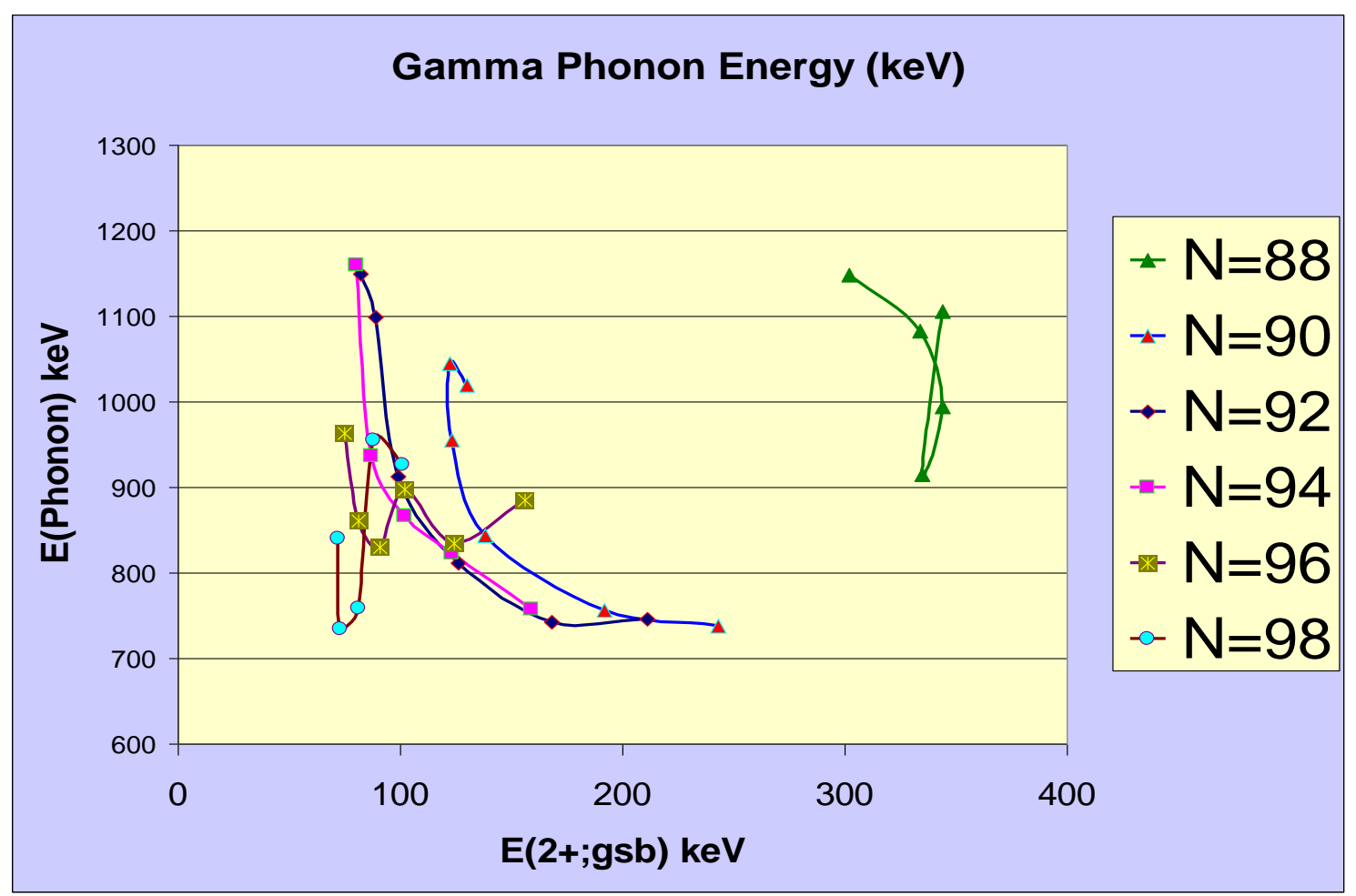

Figure 3: Phonon energies $\hbar \omega_{\gamma}$ in $\mathrm{keV}$ versus the level energy of the first excited $2_{1}{ }^{+}$level in the ground state band for deformed nuclei with neutron numbers $\mathrm{N}=88$ to 98 . The deformation increases as the $2_{1}{ }^{+}$level energy decreases. Nuclei with $\mathrm{N}=88$ have a ground state energy that is abnormally lowered with respect other levels in the ground state bands. However the range of $\hbar \omega_{\gamma}$ for $\mathrm{N}=88$ is not remarkably different from the other more deformed nuclei.

Table 1 Some even-even nuclei in which the $\gamma$-band has been observed above $15^{+}$.

\begin{tabular}{|c|c|c|c|c|c|c|}
\hline \multirow[t]{2}{*}{$\underline{\text { Nucleus }}$} & \multicolumn{2}{|c|}{$\underline{\text { Beam }}$} & \multicolumn{3}{|c|}{ Highest Spin Reached } & \multirow[t]{2}{*}{$\underline{\text { Reference }}$} \\
\hline & Specie & Energy $(\mathrm{MeV})$ & Yrast band & y-even & $\gamma-o d d$ & \\
\hline${ }^{104}$ Mo & ff (fiss & on fragment) & $20^{+}$ & $18^{+}$ & $17^{+}$ & [49] \\
\hline${ }^{154} \mathrm{Gd}$ & $\alpha$ & 45 & $24^{+}$ & $16^{+}$ & $17^{+}$ & {$[3]$} \\
\hline${ }^{156}$ Dy & ${ }^{12} \mathrm{C}$ & 65 & $32^{+}$ & $28^{+}$ & $27^{+}$ & [50] \\
\hline${ }^{156} \mathrm{Er}$ & ${ }^{48} \mathrm{Ca}$ & 215 & $26^{+}$ & $26^{+}$ & $15^{+}$ & [51] \\
\hline${ }^{160} \mathrm{Er}$ & ${ }^{48} \mathrm{Ca}$ & 215 & $50^{+}$ & - & $43^{+}$ & [52] \\
\hline${ }^{162} \mathrm{Dy}^{\mathrm{a}}$ & ${ }^{118} \mathrm{Sn}^{\mathrm{a}}$ & 780 Coulex. & $24^{+}$ & $18^{+}$ & $17^{+}$ & [53] \\
\hline${ }^{164} \mathrm{Dy}^{\mathrm{a}}$ & ${ }^{118} \mathrm{Sn}^{\mathrm{a}}$ & 780 Coulex. & $22^{+}$ & $18^{+}$ & $11^{+}$ & [53] \\
\hline${ }^{164} \mathrm{Er}$ & ${ }^{9} \mathrm{Be}$ & 59 & $24^{+}$ & $14^{+}$ & $19^{+}$ & [54] \\
\hline${ }^{164} \mathrm{Er}$ & ${ }^{18} \mathrm{O}$ & 70 & $24^{+}$ & $18^{+}$ & $21^{+}$ & {$[55,56]$} \\
\hline${ }^{170} \mathrm{Er}$ & ${ }^{238} \mathrm{U}$ & 1358 Coulex. & $26^{+}$ & $18^{+}$ & $19^{+}$ & [57] \\
\hline${ }^{180} \mathrm{Hf}$ & ${ }^{136} \mathrm{Xe}$ & 750 Coulex. & $18^{+}$ & $16^{+}$ & $13^{+}$ & [58] \\
\hline${ }^{238} \mathrm{U}$ & ${ }^{209} \mathrm{Bi}$ & $1130 \& 1330$ Coulex & $30^{+}$ & $26^{+}$ & $27^{+}$ & [59] \\
\hline
\end{tabular}


A notable feature of $\gamma$-bands is that they track the intrinsic configuration, usually the ground state that they are based on. An example of this is shown in Fig. 4 for the $\gamma$-band in ${ }^{156}$ Dy [50]. Here the $\gamma$-band tracks the ground state configuration up to its highest spin of $28^{+}$. The aligned band in ${ }^{156} \mathrm{Dy}$, which causes a back-bend in the band based on the $0_{2}{ }^{+}$state [60], shows no sign of any interaction with the $\gamma$-band. The $\gamma$-band has a small signature splitting at higher spins.

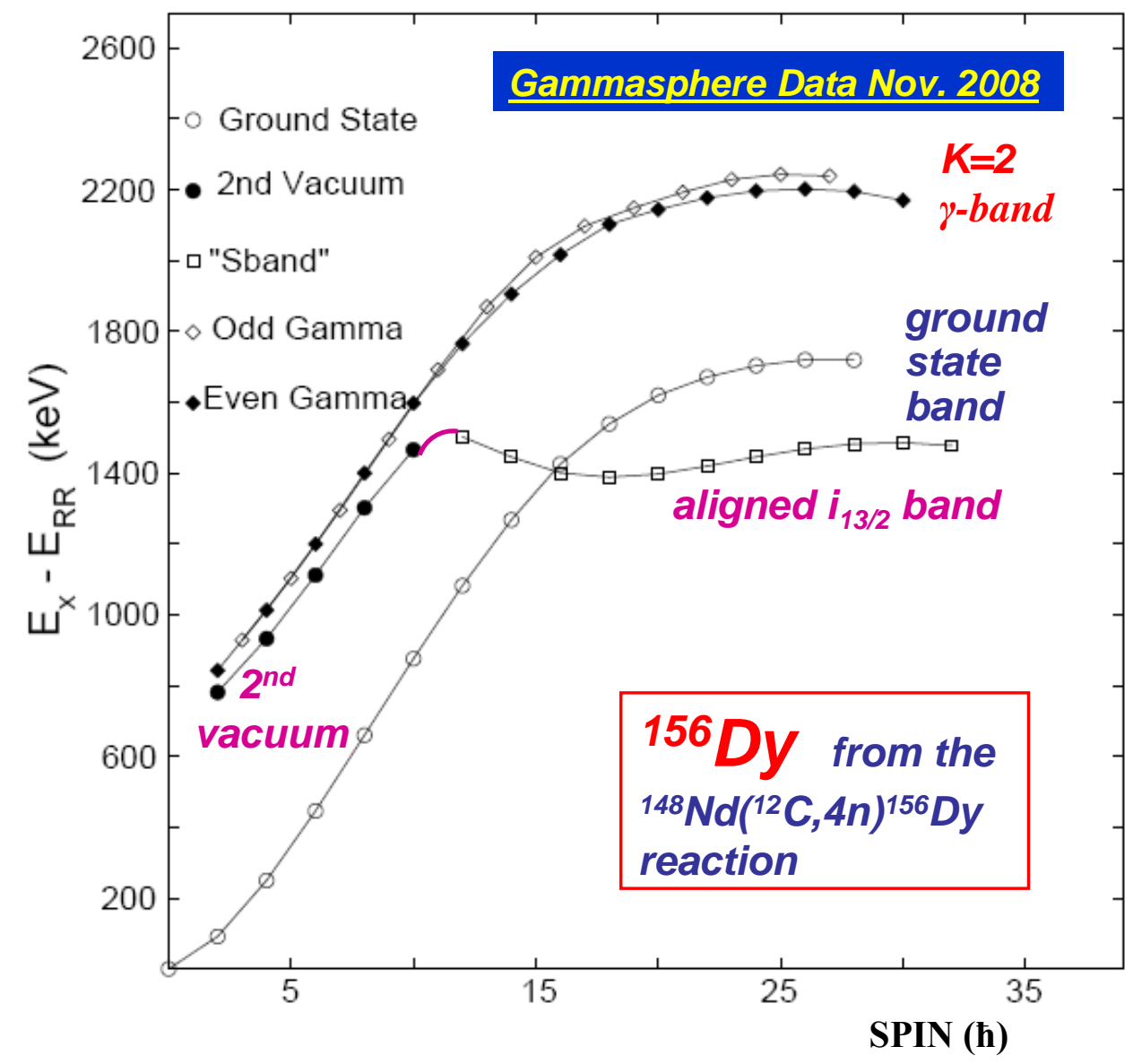

Figure 4: The excitation energies, minus a rotational energy, of positive parity bands in ${ }^{156} \mathrm{Dy}$ [50].

The question then arises, are there $\gamma$-vibrations based on the aligned configurations? Indeed such a crossing has been observed in ${ }^{164} \mathrm{Er}[55,56]$ The experimental data showing this crossing is shown in Fig. 5 together with a calculation based on the rotational-alignment model. The data shows that the ground state band is crossed by the usual aligned- or S-band. The $\gamma-$ band tracks the ground state band at lower spins and then aligns to follow the aligned yrast states. It can be seen from Fig.5 that the alignment in the $\gamma$-band will come at a slightly lower spin and hence a slightly lower rotational frequency than for the yrast states. This is because the band crossing is just from two different configurations; the $\boldsymbol{\gamma}$-band built on the ground state $\mid \mathbf{0}_{\mathbf{1}}{ }^{+}$ $>$ is crossed by a band which is the $\gamma$-band built on the aligned band $\left|\mathbf{0}_{1}{ }^{+}+\left(\mathbf{i}_{13 / 2}\right)^{2}\right\rangle$. An even more spectacular example of a $\gamma$-band built on sequential alignments has been found in ${ }^{160} \mathrm{Er}$ and is shown in Fig.6 from the very recently published work of Ollier et al. [52]. Only the odd spin members of the $\gamma$-band are seen, but they extend up to spin $43^{+}$and track the yrast states 
around both the $\mathbf{v}\left(\mathbf{i}_{13 / 2}\right)^{2}$ neutron alignment and then around the $\pi\left(\mathbf{h}_{11 / 2}\right)^{\mathbf{2}}$ proton alignment (second back-bend). Again the data indicates that the crossings of the $\gamma$-band come at slightly lower spins and frequencies than in the yrast states. The data in Fig. 6 is quite remarkable. It shows that on every intrinsic or aligned configuration a $\boldsymbol{\gamma}$-band is built with an added $\boldsymbol{K}+\mathbf{2}$ quantum number. Whatever the $\gamma$-bands are built with, it does not seem to be affected by the configurations causing the alignments. Calculations of $\gamma$-vibrations built on the aligned configuration have been made by Matsuzaki [61] for ${ }^{182} \mathrm{Os}$, but unfortunately for a band that was later found to be something else [62].

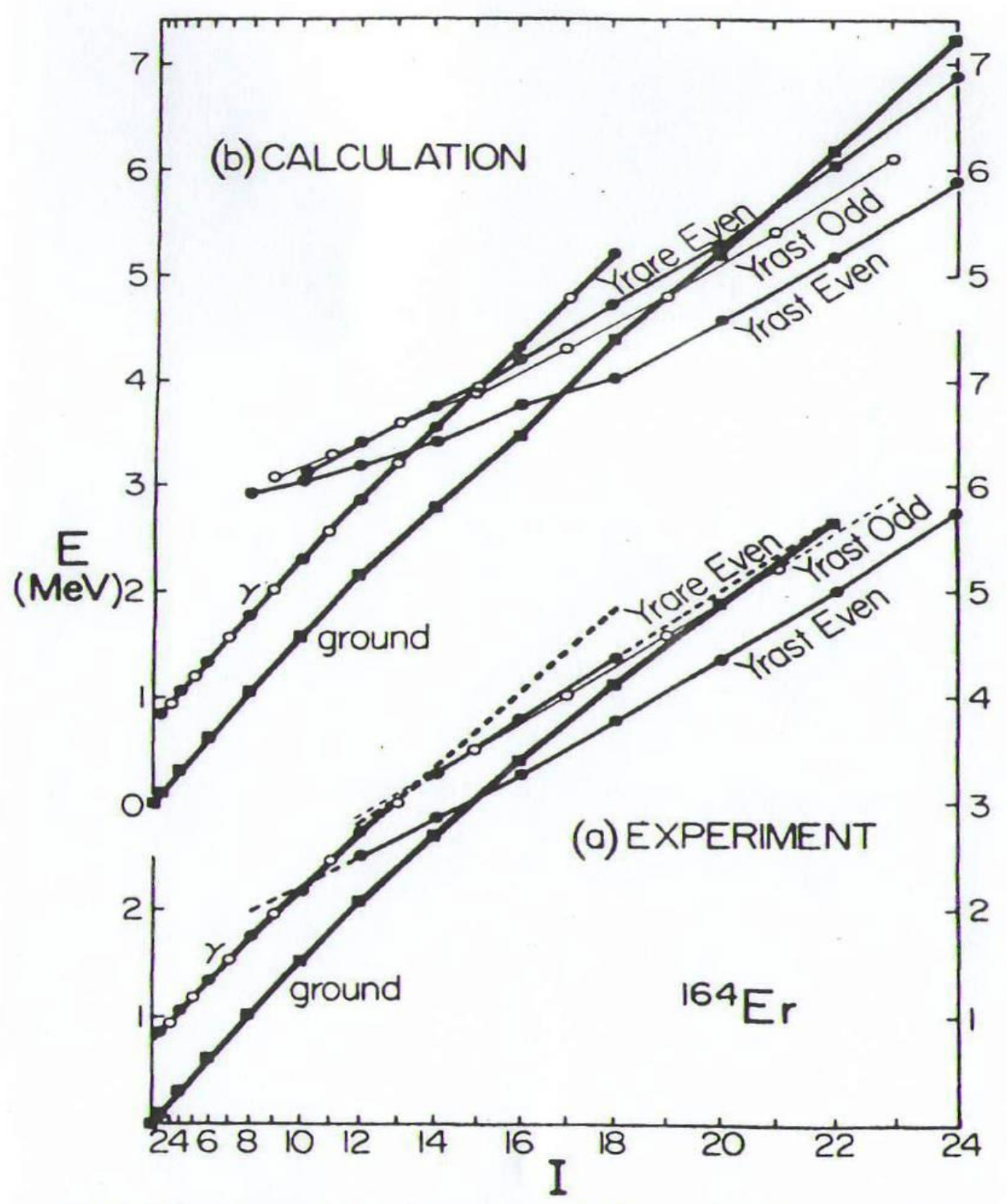

Figure 5: Plot of the level excitation energies for positive parity bands in ${ }^{164} \mathrm{Er}$ against spin on an I(I+1) scale [56]; (a) experiment and (b) the rotation-aligned model. The solid circles correspond to even-spin states and the open circles to odd-spin states. 

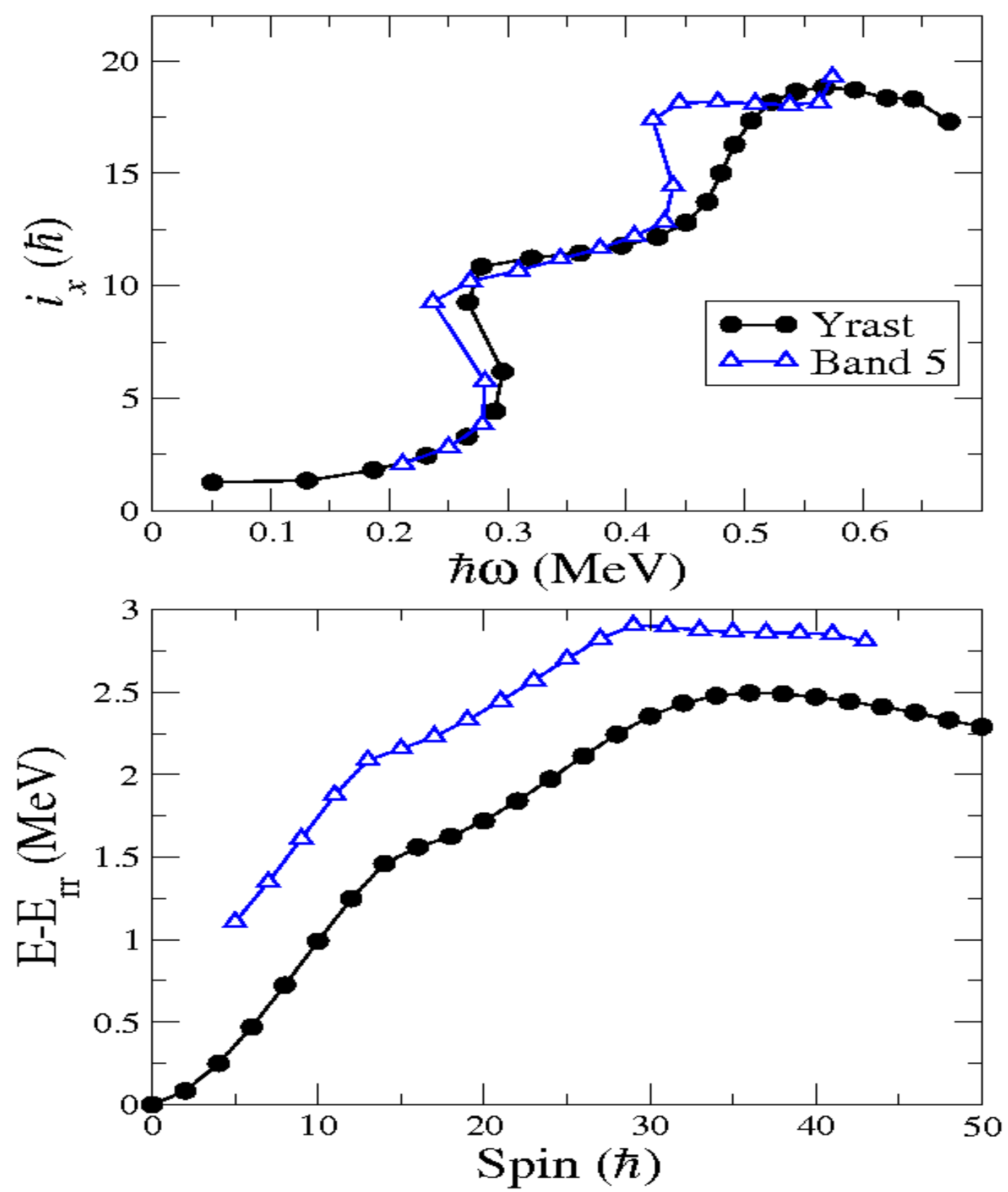

Figure 6: The position of the odd-spin $\gamma$-band (band 5) with respect to the yrast states in ${ }^{160} \mathrm{Er}$ [52]; (upper) the aligned angular momentum as a function of rotational frequency, (lower) excitation energy, minus a rigid rotor energy, against spin.

It is very interesting to note that in [52] the even members of the $\gamma$-band in ${ }^{160} \mathrm{Er}$ have not been observed. Presumably they are shifted to higher energies by some interaction and/or mixing with other even-spin low- $\boldsymbol{K}$ bands. This indicates that there should be considerable signature splitting in this $\gamma$-band. A measure of signature splitting is given by;

$$
\boldsymbol{S}(\boldsymbol{I})=\frac{[E(I)-E(I-1)]-[E(I-1)-E(I-2)]}{E\left(2_{1}^{+}\right)}
$$


In ${ }^{156} \mathrm{Er}$ the $\gamma$-band is seen up to spin $26^{+}$, by which spin the configuration includes the aligned $\boldsymbol{v}\left(\mathbf{i}_{13 / 2}\right)^{2}$ neutrons [51]. But in this case, less deformed then ${ }^{160} \mathrm{Er}$, the aligned $\gamma$-band is only observed for even spins. The $\gamma$-band in ${ }^{156} \mathrm{Er}$ shows a marked signature splitting, as shown in Fig. 7 where the level energies and the quantity $\boldsymbol{S}(\boldsymbol{I})$ are plotted as a function of spin. This signature splitting is usually interpreted as an effect of permanent $\gamma$ deformation ([51] and references therein).

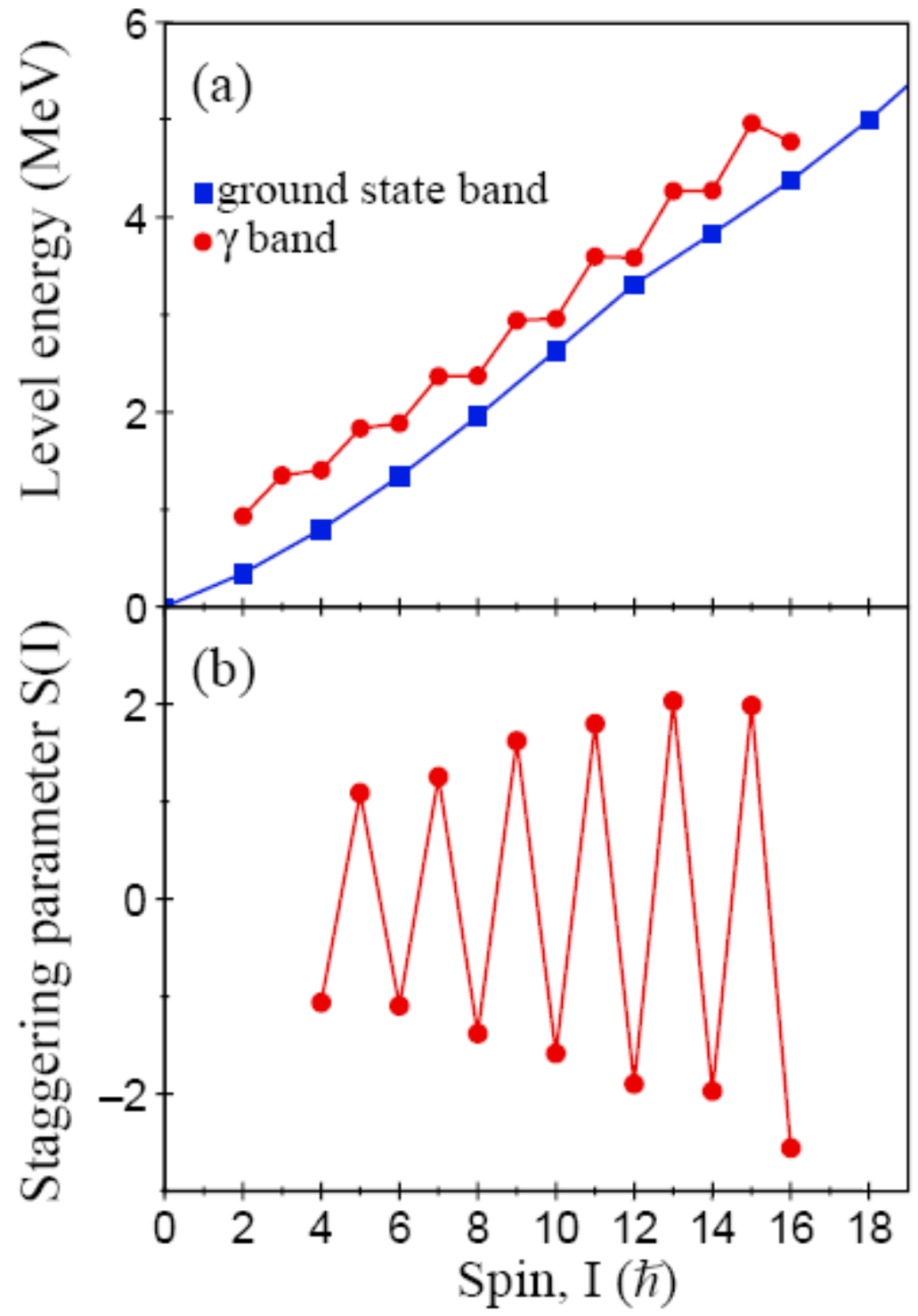

Figure 7: (a) The ground state and $\gamma$-band energies and (b) the signature splitting parameter $\boldsymbol{S}(\boldsymbol{I})$ as a function of the spin $\boldsymbol{I}$.

There have been many studies of the transition strengths of out-of-band transitions from $\gamma$-bands to their ground state bands. In Fig. 8 we show the B(E2) strengths, in Weisskopf units (W.u.), from the $\boldsymbol{K}^{\pi}{ }_{\gamma}=2^{+}$band head to the ground state of a selection of nuclei. It can be seen that the transition strength averages about $5 \mathrm{~W}$.u. indicating that the transition is not very strongly collectively. 


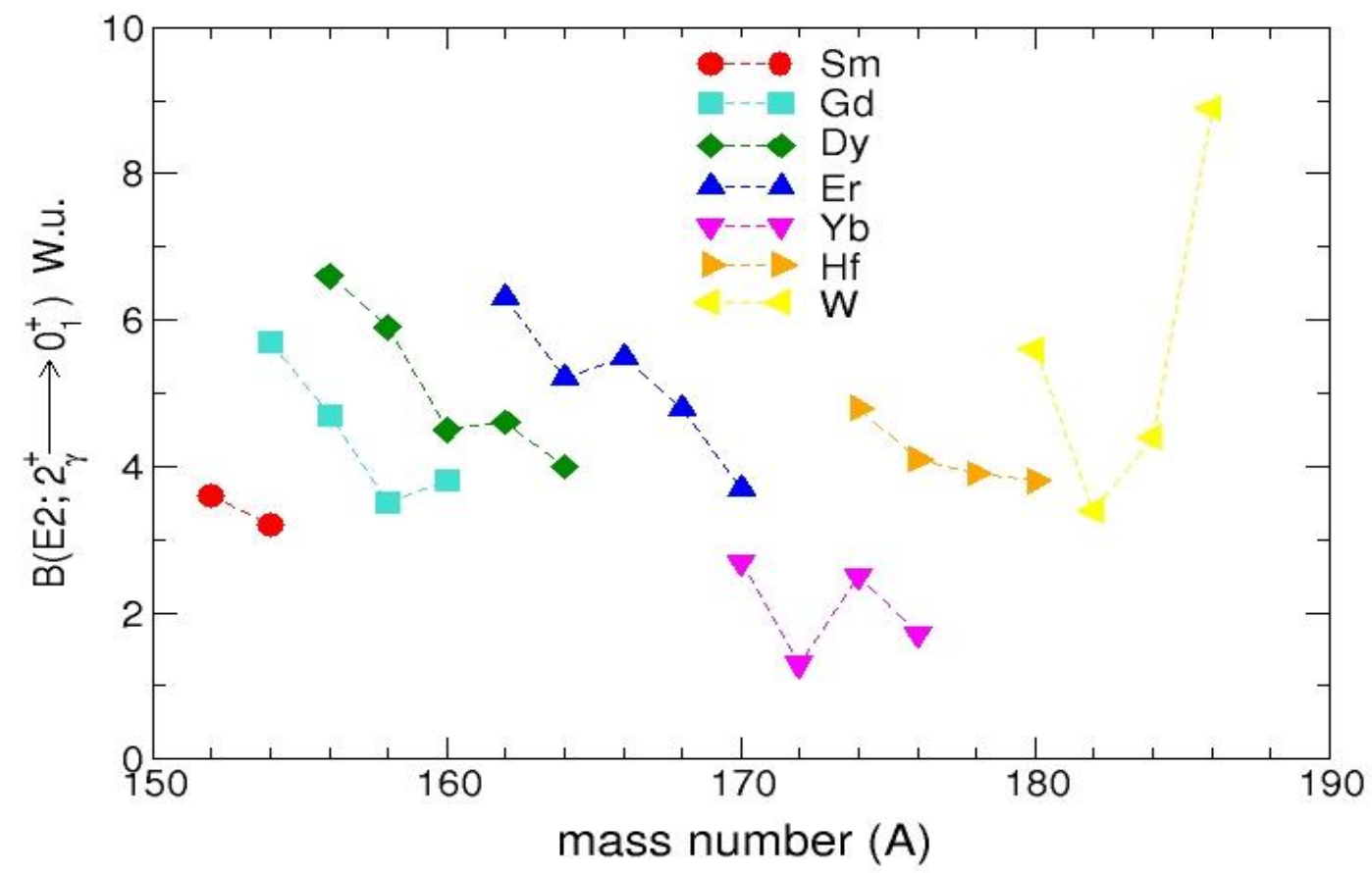

Figure 8: Systematics of the strength of the E2 transitions from the $\mathbf{2}_{\gamma}{ }^{+} \gamma$-bandhead to the ground state in a series of deformed nuclei.

\section{Odd Nuclei; Coupling of Single Particle States to $\gamma$-Vibrations}

One strong test of any theory of $\gamma$-vibrations is the experimental evidence of how single particle states couple to the core $\gamma$-vibration. Each single particle state with Nilsson quantum number $\boldsymbol{\Omega}$ can couple to the core $\boldsymbol{K}^{\pi}=2^{+}$either in a parallel mode to give $\boldsymbol{K}_{>}=\boldsymbol{\Omega}+\boldsymbol{2}$ or in an anti-parallel mode to give $\boldsymbol{K}_{<}=|\boldsymbol{\Omega}-2|$. There can be a splitting of the band heads of these two bands which will give information on the particle-vibration interaction. Clearly the band with $\boldsymbol{K}$, will usually be nearer yrast and therefore easier to detect in (HI,xn) reactions. However the $\boldsymbol{K}_{<}$ band can be found, when $\boldsymbol{K}_{<}$is small, in experiments such as (n, $\left.\gamma\right)$ and (n,n' $\left.\gamma\right)$ experiments [63].

The most complete data sets on the coupling of the ground state nucleon in an odd nucleus to a core $\gamma$-vibration are Coulex experiments [64] on ${ }_{67}^{165} \mathrm{Ho}_{98}$ and ${ }_{68}^{167} \mathrm{Er}_{99}$, which share the core nucleus ${ }_{68}^{166} \mathrm{Er}_{98}$, and fission fragment spectroscopy [65] on the trio ${ }_{41}^{103} \mathrm{Nb}_{62},{ }_{42}^{104} \mathrm{Mo}_{62}$ and ${ }_{42}^{105} \mathrm{Mo}_{63}$.

In Fig. 9 we show the excitation energies of the ground state, $\boldsymbol{K}_{>}$and $\boldsymbol{K}_{<}$bands in ${ }_{67}^{165} \mathrm{Ho}_{98}$. The energies of the $\boldsymbol{K}_{>}$and $\boldsymbol{K}_{<}$band heads are 689 and $515 \mathrm{keV}$ respectively, giving a splitting of $174 \mathrm{keV}$. The core $\gamma$-bandhead in ${ }_{68}^{166} \mathrm{Er}_{98}$ is at $786 \mathrm{keV}$. It can be seen that the $\boldsymbol{K}$, and $\boldsymbol{K}_{<}$bands again track the ground state band and that there is no significant signature splitting in any of the 3 bands. In Fig. 10 the excitation energies, minus a rigid rotor energy, for the [633]7/2 $2^{+}$ground state band in ${ }_{68}^{167} \mathrm{Er}_{99}$ and the $\boldsymbol{K}_{>}$band are shown. The $\boldsymbol{K}_{<}$band has not been observed. The $\boldsymbol{K}$, band head is at $709 \mathrm{keV}$, close to the $786 \mathrm{keV}$ of the core excitation. In this case the $[633] 7 / 2^{+}$ground state band shows significant signature splitting at higher spins, whereas the $\boldsymbol{K}$, band does not. 


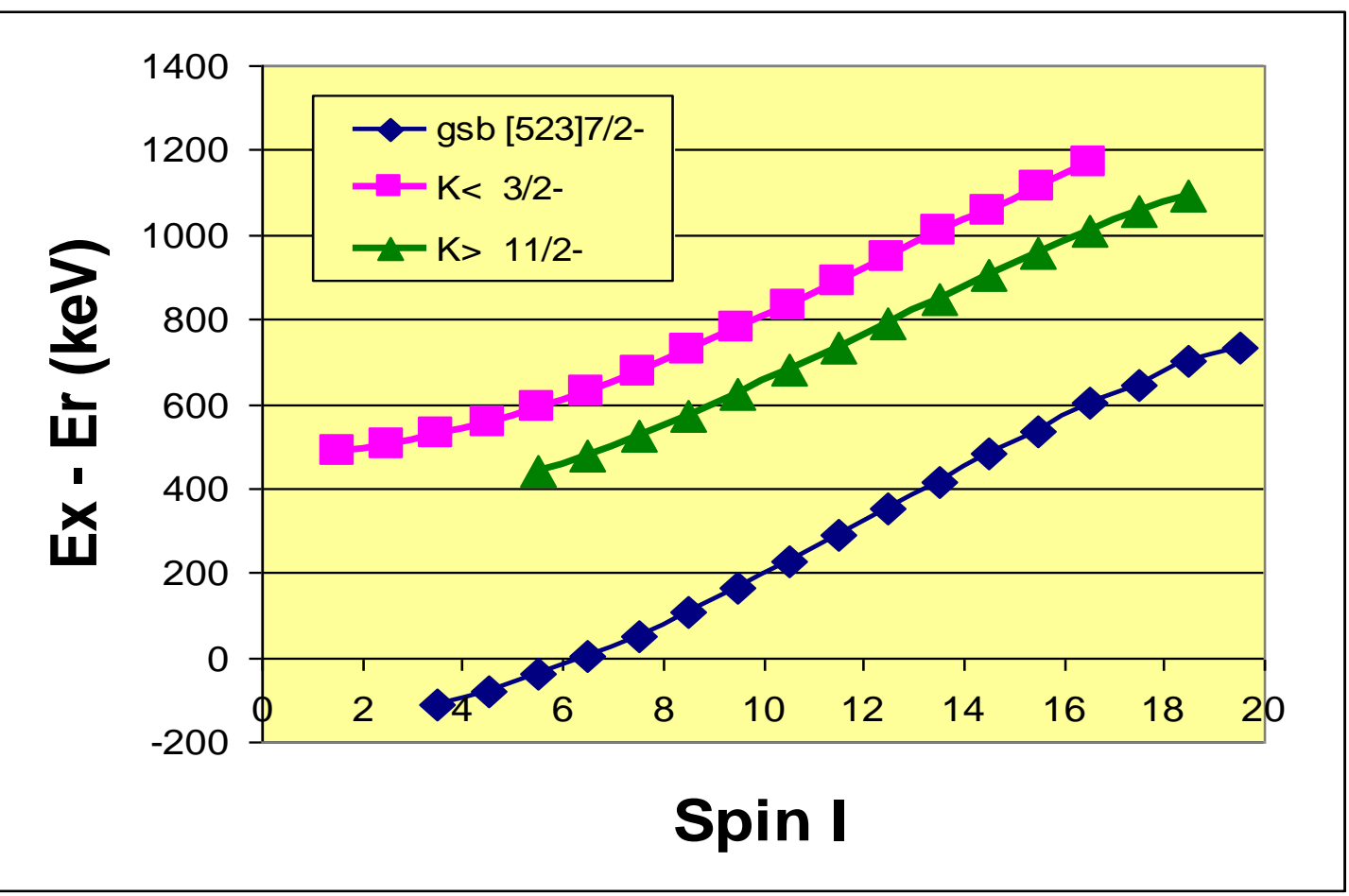

Figure 9: Excitation energies, minus a rigid rotor energy, for the [523]7/2- ground state band in ${ }_{67}^{165} \mathrm{Ho}_{98}$ and the $\boldsymbol{K}_{>}$and $\boldsymbol{K}_{<}$bands formed by coupling the [523]7/2- proton to the $\boldsymbol{\gamma}$ vibration of the ${ }_{68}^{166} \mathrm{Er}_{98}$ core [64].

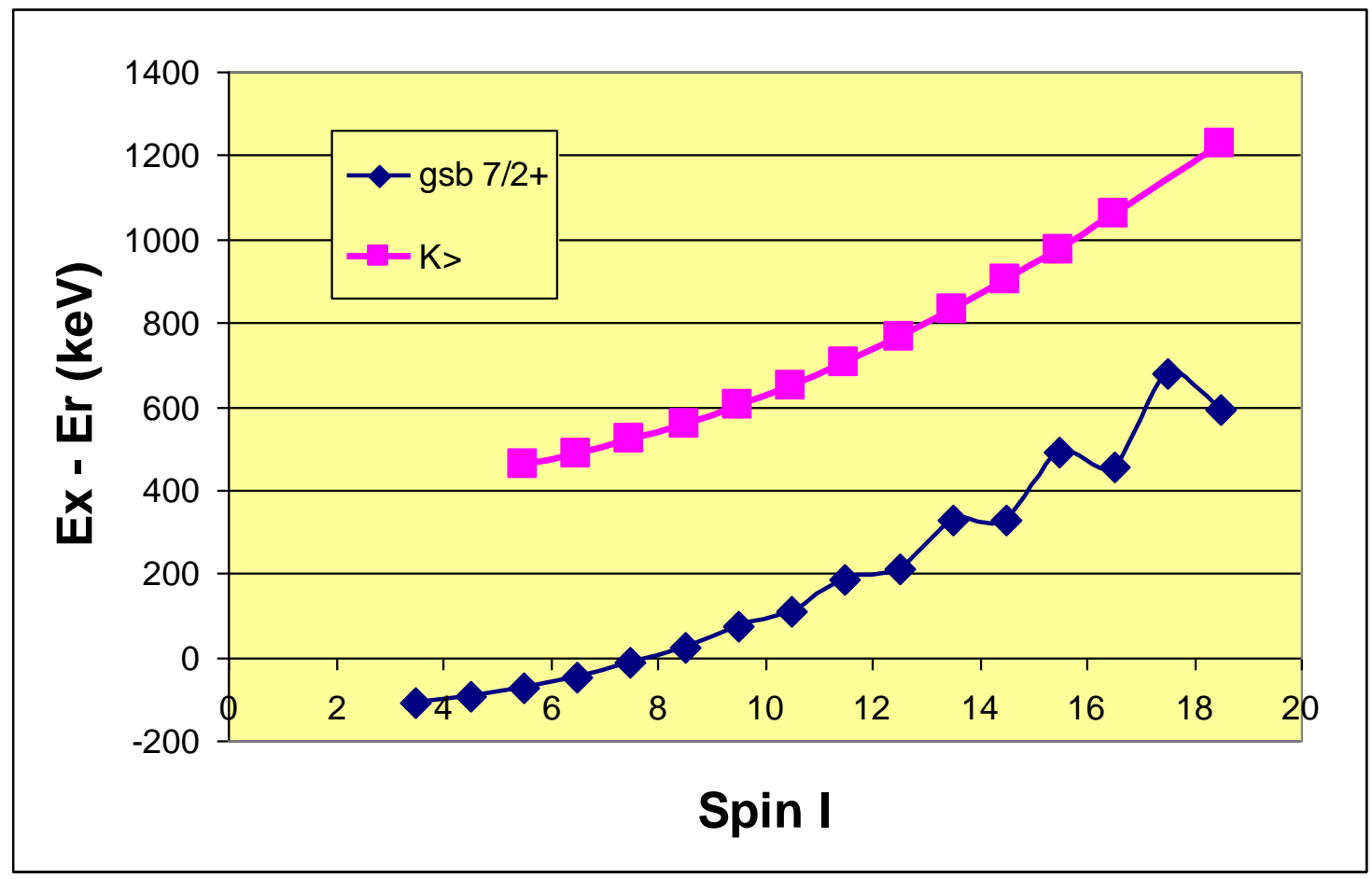

Figure 10: Excitation energies, minus a rigid rotor energy, for the $[633] 7 / 2^{+}$ground state band in ${ }_{68}^{167} \mathrm{Er}_{99}$ and the $\boldsymbol{K}$, band formed by coupling the [633]7/2+ neutron to the $\gamma$ vibration of the ${ }_{68}^{166} \mathrm{Er}_{98}$ core [64]. 
In Fig. 11 the excitation energies, minus a rigid rotor energy, are shown for the nuclei ${ }_{41}^{103} \mathrm{Nb}_{62},{ }_{42}^{104} \mathrm{Mo}_{62}$ and ${ }_{42}^{105} \mathrm{Mo}_{63}$. In this case we show the ground bands, the $\boldsymbol{K}$, bands and $\boldsymbol{K}^{\pi}=4^{+}$bands that the authors [65] suggest are a double $\gamma$-vibration. Again all the excited bands shown track the ground bands. The excited bands show no signature splitting, whereas the odd proton nucleus ${ }_{41}^{103} \mathrm{Nb}_{62}$ shows some splitting and in the odd neutron nucleus ${ }_{42}^{105} \mathrm{Mo}_{63}$ the signature splitting is considerable.
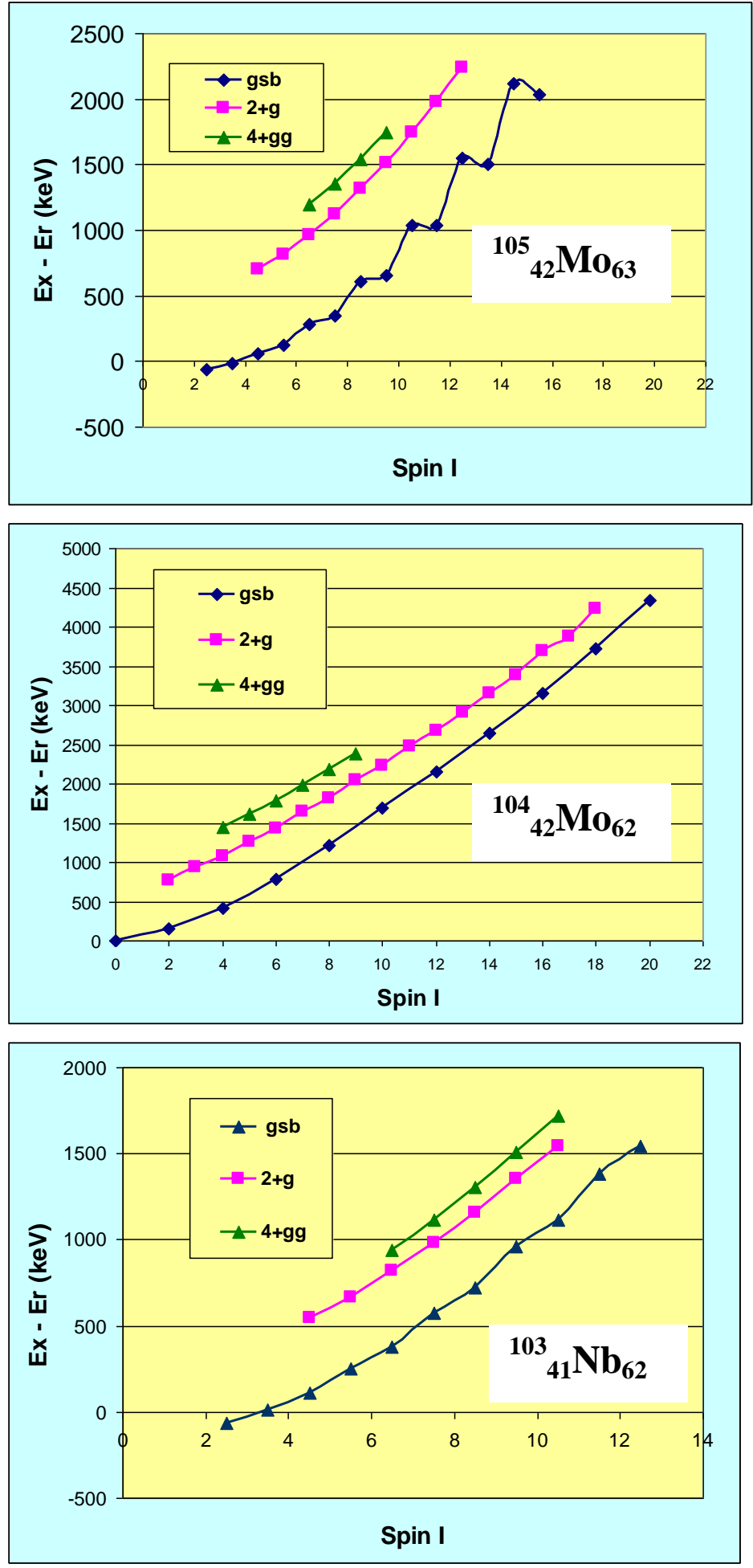

Figure 11: Excitation energies, minus a rigid rotor energy, are shown for the nuclei $\quad{ }_{103}{ }_{41} \mathrm{Nb}_{62},{ }_{104}^{104} \mathrm{Mo}_{62}$ and ${ }_{42}^{105} \mathrm{Mo}_{63}$. In this case the ground bands (gsb), the $\boldsymbol{K}$, bands $\left(2^{+} \mathrm{g}\right)$ and $\boldsymbol{K}^{\pi}=\mathbf{4}^{+}$ bands $\left(4^{+} \mathrm{g}\right)$, that the authors suggest are a double $\gamma$ vibration, are shown. 


\section{Conclusions}

We can summarise the experimental information on $\gamma$-bands as;

(i) . $\mathrm{B}(\mathrm{E} 2)$ decays out-of-band to ground state band have $\Delta \mathrm{K}=2$ and hence no M1 strength. Any E2/M1 mixing ratios $\delta>>1$.

(ii) $\quad$ M1 in-band transitions $\Delta \mathrm{J}=1$ are very weak or zero showing that $\boldsymbol{g}_{K} \approx \boldsymbol{g}_{\boldsymbol{R}}$ for $\boldsymbol{\gamma}$-bands.

(iii) Transfer reactions (pick-up \& stripping) are near zero or very weak. There are rare exceptions when there are levels on the accessible side of the Fermi level with $\boldsymbol{\Delta} \boldsymbol{K}=\boldsymbol{2}$.

(iv) Some bands are Signature split, others are not. Is this connected to $\gamma$-deformation ??

(v) It is very impressive that ALL $\gamma$-bands track their intrinsic configuration.

(vi) There are very few examples of $\mathrm{K}>/ \mathrm{K}<$ splitting in odd nuclei.

It is fairly clear that more data on $\gamma$-vibrations built on different intrinsic configurations would help. Also to trace the $\gamma$-bands in nuclei with different behaviours of their yrast bands. More examples of both $\boldsymbol{K}$, and $\boldsymbol{K}_{<}$bands in the same nucleus would also help. Experimentally Coulomb excitation is a good way of connecting to real collective structures and it keeps the spectra obtained less complicated. Otherwise the big arrays, such as Gammasphere etc, should be used to study yrare structures with lower angular momentum reactions than usual. It is not yet clear to us if the " $\gamma$-vibrational" bands are just a $\boldsymbol{K}^{\pi}=2^{+}$projection of the zero point motion on the symmetry axis, or if they are more of a traditional Boson or Phonon? What is rather evident is that there is a $\gamma$-vibration built on every intrinsic state. We are impressed by the relative successes of RPA and TPSM calculations and we are forced, by the experimental data, to regard non-microscopic models as no use at all!

Unlike the phantom $\beta$-vibrations, $\gamma$-vibrations are a REAL collective motion!

\section{Acknowledgements}

We would like to thank all our many colleagues in many institutions for many inspiring discussions and for considerable help. JFSS would like to thank the Joyce Frances Adlard Cultural Find for financial support and also the organisers of Bormio 2011.

\section{References}

[1] Å. Bohr and B. R. Mottelson, "Nuclear Structure, Vol. II”, Singapore: World Scientific, 1998, pp. 363.

[2] P. E. Garrett, J. Phys. G (London) 27, R1 (2001).

[3] J. F. Sharpey-Schafer et al, Eu. Phys. J. A47 (2011) 5 and 6.

[4] Å. Bohr, K. Dan. Vid. Selsk. Mat.-Fys. Medd. 26 (1953) No.14

[5] Å. Bohr and B.R. Mottelson, K. Dan. Vid. Selsk. Mat.-Fys. Medd. 27 (1953) No. 16.

[6] L. Próchniak and S. G. Rohoziński, J. Phys. G36 (2009) 123010.

[7] J. P. Davidson, Collective Models of the Nucleus, New York and London, Academic, 1968, p39.

[8] W. Greiner and J. A. Maruhn, Nuclear Models (Springer 1996) p158/9 
[9] D. R. Bès, K. Dan. Vid. Selsk. Mat.-Fys. Medd. 33 (1961) No. 2.

[10] D. R. Bès, Nucl. Phys. 49 (1963) 544

[11] D. R. Bès, P. Federman, E. Maqueda and A. Zucker, Nucl. Phys. 65 (1965) 1

[12] V. G. Soloviev, Nucl. Phys. 69 (1965) 1

[13] F. A. Gareev et al., Sov. J. Part. Nucl. 4, 148-195 (1973).

[14] D. Jansson and I. N. Mikhailov, Nucl. Phys. A318 (1979) 390.

[15] V. G. Soloviev and N. Yu. Shirikova, Z. Phys. A301 (1981) 263.

[16] R. Piepenbring and M. K. Jammari, Nucl. Phys. A481(1988) 81.

[17] Steven A. Stotts and Taro Tamura, Phys. Rev. C40 (1989) 2342.

[18] J. P. Delaroche et al., Phys. Rev. C50 (1994) 2332.

[19] F. Iachello, Phys. Rev. Lett. 85 (2000) 3580

[20] E. A. McClutchan et al., Phys. Rev. C76 (2007) 024306.

[21] E. R. Marshalek and J. Wesner, Phys. Rev. C2 (1970) 1682.

[22] E. S. Hernández and A. Plastino, Z. Phys. 268 (1974) 337 and A273 (1975) 273.

[23] J. L. Egido, H. J. Mang and P. Ring, Nucl. Phys. A339 (1980) 390.

[24] Yoshifumi R. Shimitzu and Kenichi Matsuyanagi, Prog. Th. Phys. 72 (1984) 799.

[25] K. Hara and Y. Sun, Int. J. Mod. Phys. E4 (1995) 637.

[26] J. A. Sheikh and K. Hara, Phys. Rev. Lett. 82 (1999) 3968.

[27] Yang Sun et al., Phys. Rev. C61 (2000) 064323.

[28] J. A. Sheikh et al., Phys.Rev. C77 (2008) 034313

[29] J.A. Sheikh et al., Nucl. Phys. A824 (2009) 58.

[30] T. Nikšić et al., Phys. Rev. Lett. 99 (2007) 092502

[31] Z. P. Li et al., Phys. Rev. C79 (2009) 054301

[32] W. N. Shelton and C. E. Watson, Phys. Lett. 22 (1966) 648

[33] Ch. Hinke et al., Eur. J. Phys. A30 (2006) 357

[34] D. E. Nelson, D. G. Burk, J. C. Waddington and W. B. Cook, Can. J. Phys. 51 (1975) 200

[35] D. G. Burk, J. C. Waddington and O. P. Jolly, Nucl. Phys. A688 (2001) 716

[36] C. R. Hirning and D. G. Burk, Can. J. Phys. 55(1977) 2288

[37] S. J. Freeman et al., Nucl. Phys. A554 (1993) 333

[38] S. J. Freeman et al., Nucl. Phys.A552 (1993) 10

[39] Th. W. Elze, J. S. Boyno and J. R. Huizenga, Nucl. Phys. A187 (1972) 473 
[40] D. G. Fleming et al., Phys. Rev. C8 (1973) 806

[41] M. H. Mortensen, R. R. Betts and C. K. Brockelman, Phys. Rev. C21 (1980) 2275

[42] D. Bucurescu et al., Phys. Rev. C73 (2006) 064309

[43] H.-F. Wirth et al., Phys. Rev. C69 (2004) 044310

[44] Casten et al., Phys. Lett. 40B (1972) 333

[45] M. A. M. Shahabuddin et al., Nucl. Phys. A340 (1980) 109

[46] J. H. Bjerregaard et al., Nucl. Phys. 86 (1966) 145

[47] K. Schreckenbach and W. Gelletly, Phys.Lett. 94B (1980) 298

[48] I. Alfter, E. Bodenstedt, W. Knichel and J. Schüth, Nucl. Phys. A635 (1998) 273

[49] Nucl. Data Sheets, 108 (2007) 2035

[50] S N T Majola, D Hartley, L L Riedinger et al, to be published

[51] J M Rees et al., Phys. Rev. C83 (2011) 044314

[52] J Ollier et al., Phys. Rev. C83 (2011) 044309

[53] C Y Wu et al., Phys. Rev. C64 (2001) 064317

[54] O C Kistner, A W Sunyar and E der Mateosian, Phys. Rev. C17 (1978) 1417

[55] N R Johnson et al., Phys. Rev. Lett. 40 (1978) 151

[56] S W Yates et al., Phys. Rev. C21 (1980) 2366

[57] C Y Wu et al., Phys. Rev. C61 (2000) 021305(R)

[58] E Ngijoi-Yogo et al., Phys. Rev. C75 (2007) 034305

[59] D Ward et al., Nucl Phys A600 (1996) 88

[60] D Ward, R L Graham, J S Geiger and H R Andrews; Phys. Lett. 44B (1973) 39

[61] Masayuki Matsuzaki, Nucl. Phys. A509 (1990) 269

[62] P Chowdhury, private communication.

[63] H H Schmidt et al., J. Phys. G12 (1986) 411

[64] G Gervais et al., Nucl. Phys. A624 (1997) 257

[65] Jian-Guo Wang et al., Nucl. Phys. A834 (2010) 94c 